\title{
Systems-level evidence of transcriptional co-regulation of yeast protein complexes
}

\author{
J. William Lee ${ }^{1}$, Tomasz Zemojtel ${ }^{2}$, Eugene Shakhnovich ${ }^{1}$
}

${ }^{1}$ Dept. of Chemistry and Chemical Biology, Harvard University, Cambridge, MA 02138 , USA; ${ }^{2}$ Dept. of Computational Molecular Biology, Max Planck Institute for Molecular Genetics, 14195 Berlin, Germany

Protein complexes are functional units in the cell whose formation is regulated by a number of mechanisms. Previous studies have suggested that transcriptional co-regulation of protein components play a minor role in assembly of individual complexes. Recent developments in experimental approaches and bioinformatic analyses offer an opportunity to investigate the cell at a global or systems level with better quality. Here we show that, using genome-/proteome-wide yeast data of protein complexes and protein-DNA interactions in normal growth conditions, a contribution of transcriptional co-regulation to global formation of protein complexes is statistically significant at a systems level. A significant fraction of proteins that form complexes shares common transcription factors on a global scale. We provide evidence for the significant contribution by quantifying transcriptional co-regulation of complexes as a global topological pattern of an integrated network. Our co-regulation measure for a complex takes into account both the absolute fraction of co-regulated protein components and their probabilistic scores from the hypergeometric distribution for all transcription factors. Biological relevance is examined by functional analysis of highly co-regulated complexes. Our finding indicates that there exists linear causality at a systems level between transcriptional co-regulation of genes and formation of multi-protein complexes in the unicellular organism.

\section{References}

[1] Gavin et al. (2006). Proteome survey reveals modularity of the yeast cell machinery, Nature 440: $631-636$

[2] Krogan et al. (2006). Global landscape of protein complexes in the yeast Saccharomyces cerevisiae., Nature 440: 637 - 643

[3] Reguly et al. (2006). Comprehensive curation and analysis of global interaction networks in Saccharomyces cerevisiae, Journal of Biology 5(4): 11

[4] Harbison et al. (2004). Transcriptional regulatory code of a eukaryotic genome, Nature 431(7004): 99-104

[5] Simonis et al. (2004). Transcriptional regulation of protein complexes in yeast, Genome Biol 5: R33

[6] Yeger-Lotem et al. (2004). Network motifs in integrated cellular networks of transcriptionregulation and protein-protein interaction, Proc Natl Acad Sci USA 101: 5934 - 5939

[7] Zhang et al. (2005). Motifs, themes and thematic maps of an integrated Saccharomyces cerevisiae interaction network, Journal of Biology 4(2): 6 